\title{
Reconstrucción del enfoque del aumento y generación del efectivo para la Pyme de manufactura
}

Igor Rivera González

Unidad Profesional Interdisciplinaria de Ingeniería y Ciencias Sociales y Administrativas (UPIICSA), Instituto Politécnico Nacional

iariverag@ipn.mx

Juan Morúa Ramírez Covarrubias Consultores y de High Performance S.A. jmorua@prodigy.net.mx

\section{Resumen}

Este artículo parte de la hipótesis de que el aumento continuo del efectivo es el objetivo principal dentro de las pequeñas y medianas empresas privadas de manufactura ${ }^{3}$. Nuestra finalidad es realizar una propuesta teórica acerca de cómo definir la paramétrica referida al aumento del efectivo en un periodo a través del análisis de la forma en que se genera el efectivo dentro de las pequeñas y medianas empresas (Pyme) de trasformación; para ello, se resalta la importancia de tres elementos clave que intervienen en la generación del efectivo: a) el proceso de transformación, b) la optimización del flujo de materiales y de información y c) la creación de valor.

Palabras clave: aumento de efectivo, proceso de generación de efectivo, teoría trasformación-flujo-valor, teoría de restricciones. 


\title{
Rebuilding the cash increase and generation approach for manufacturing SME's
}

\begin{abstract}
This article is based on the hypothesis that continuous cash growth has been a principal target for small and medium manufacturing companies. We make a theoretical proposal about how to define the metrics used for the cash increase in a period of time, through the analysis of the way cash is generated, highlighting the importance of three key elements for cash generation: a) the transformation process, b) the optimization of materials and information flow, c) and value creation.
\end{abstract}

Keywords: cash growth, cash generation process, theory of transformation-flow-value, theory of constraints.

\section{Introducción}

En el proceso de toma de decisiones dentro de las empresas es importante determinar dos elementos clave: el primero es la meta que la empresa desea alcanzar, la cual va a ser un objetivo constante desde su creación; el segundo son las personas que mediante los procesos de pensamiento establecerán las estrategias por seguir para alcanzar su propósito. Para muchos autores la principal meta de las empresas privadas es el aumento y la generación de efectivo (Goldratt, 1992). Esta forma de ver a la empresa como un ente económico-financiero lo han establecido numerosos autores a través del tiempo en diversas áreas como la economía (Galbraith, 1999; Baca, 2000; Taylor, 2000), la ingeniería industrial (Mather, 1988; Koskela, 2000), la administración (Sallenave, 1997; Ochoa, 2002), entre otras. Estos autores concluyen que el aumento del efectivo es una de las principales finalidades de la mayoría de la Pyme, y va unido con la obtención de utilidades y de un buen retorno de inversión (Goldratt, 1992; Brigharn, 2006; Mather, 1988).

Para la realización de nuestro artículo tomamos como base la hipótesis del aumento de efectivo, que es uno de los principales objetivos de las pequeñas y medianas empresas (Pyme). Cuando se habla de aumento y generación de efectivo se dan por hecho muchas consideraciones obvias que requieren la atención constante de los integrantes de la empresa; por ello, decimos estudiar este proceso para entender y hacer una propuesta de cómo alinear estos dos elementos: por una parte, el proceso de aumento y generación de efectivo; por otra, los procesos de pensamiento del dirigente. 
Asimismo, la inquietud de comprender la forma en cómo se aumenta y genera el efectivo proviene de la experiencia y observación participativa que hemos desarrollado dentro de la Pyme en México, donde los pensamientos de los integrantes de las mismas y la manera en cómo se aumenta y genera el efectivo no se encuentran unificados. El mismo Galbraith (1999) ya mencionaba que "el primer requisito para la comprensión de la vida económica de la empresa [...] es lograr una visión clara de la relación existente entre los hechos y las ideas". Es por eso que para uniformar el criterio un primer requisito no sólo es definir y difundir la misión y visión de la empresa (Sink, 1999), sino también es importante conocer en forma particular la manera en cómo se aumenta y se genera el efectivo dentro de la misma.

El objetivo de este artículo es establecer teóricamente la forma en cómo se aumenta el efectivo dentro de las empresas, estableciéndolo mediante el cumplimiento de cuatro condiciones necesarias ${ }^{1}$ : 1) la existencia de flujo de efectivo, 2) que el efectivo creado por el proceso de generación dentro del sistema sea mayor que el efectivo gastado por las operaciones, 3) la existencia de disponibilidad de efectivo, y 4) que los inventarios se mantengan o disminuyan a través del periodo evaluado. Cabe aclarar que este artículo no se refiere a la retención o acumulación de efectivo, más bien está orientado a estudiar la manera en que la empresa hace crecer o aumentar el efectivo dentro de un periodo para, posteriormente, profundizar en la forma en cómo se genera el efectivo.

\section{Aumento y generación de efectivo: diferentes perspectivas}

Es importante establecer que existen numerosos enfoques relacionados con el aumento y la generación de efectivo: estratégicos (Porter, 1989), restrictivos (Goldratt, 1992), económicos (Galbraith, 1999), financieros (Brigharn, 2006), de marketing (Baker, 2001), entre otros. Estos enfoques están basados para empresas que mantienen grandes capitales y con amplias inversiones; sin embargo, la mayoría de ellos suelen estar lejos de la aplicación de pequeñas y medianas empresas. Es por esto que en este trabajo empezaremos revisando lo que es el aumento del efectivo y la generación del mismo desde diferentes perspectivas académicas.

\footnotetext{
${ }^{1} \mathrm{El}$ cumplimiento de estas condiciones es relativo a las condiciones de la empresa, aunque el cumplimiento de al menos tres de ellas es necesario; esto se explicará más adelante dentro del último apartado.
} 
Aunque la administración financiera ha desarrollado ampliamente el tema, en este artículo no estudiaremos la administración del efectivo, sino profundizaremos en el proceso de aumento y generación. Para la administración financiera el aumento de efectivo está relacionado con la actividad económica que realiza la empresa, por lo que la generación del efectivo es el resultado de los parámetros medidos a través de las cuentas contables (Brigharn, 2006); es decir, no profundizan en el proceso de generación intentando explicarlo, sino que más bien éste surge por la actividad de la misma, así la propuesta de aumento de efectivo se basa en las inversiones, la reducción de costos, la rápida recuperación de las cuentas por cobrar, entre otras acciones que se encuentran enmarcadas tanto fuera como dentro del proceso de generación (Johnson, 2002).

En el marketing (Baker, 2001) se mantiene un caso similar, el aumento de efectivo es resultado de la generación de la actividad económica, pero el principal motor que aumenta el efectivo está determinado por el portafolios de productos que la empresa ofrece en el mercado; la clave para su aumento es el resultado de una buena toma de decisiones en la mezcla de la venta de dicho portafolios, además de la conquista de nuevos mercados y la satisfacción de los clientes (Savall, 2008). Entre más se vendan, promuevan y desarrollen productos con mayor contribución, mayor efectivo se estará generando. Este punto de vista es válido, sin embargo, desde nuestra perspectiva, por sí sólo no conduce a la empresa a un aumento.

En el caso de los autores que se refieren a la estrategia empresarial, el resultado del aumento de efectivo está determinado por la toma de una serie de estrategias competitivas que permitan a la empresa vender los productos y servicios con una serie de características diferentes a los de la competencia, ya sea segmentando o posicionando a la empresa en nichos de mercado. Para Porter (1989), el proceso de generación está basado en la denominada cadena de valor, la cual está representada en una serie de actividades que en su conjunto forman no sólo parte de la generación de efectivo, sino también de una ventaja competitiva. Los autores de estrategia empresarial se basan constantemente en un enfoque de servicio, manteniendo como una de las principales actividades el lograr la satisfacción de los clientes (Koskela, 2000).

Para la economía (Taylor, 2000), el aumento de efectivo y la generación del mismo descansa en dos imperativos: el capital y el trabajo; es decir, la primera traducida en elementos monetarios, o herramientas y maquinaria; la segunda, como mano de obra encargada de contribuir al proceso de transformación (Savall, 2008); por 
lo tanto, estos dos elementos son necesarios para el aumento y generación de más efectivo.

Para la ingeniería, el proceso de aumento de efectivo está dado por las mejoras en el proceso de transformación, ya sea en cuestiones técnicas como maquinaria y herramienta, o en el aumento de las habilidades de los trabajadores. Para ello es necesario un aumento en la productividad. Smith (1979) da un aporte importante a este renglón cuando en 1776 escribe sobre la causa de la naturaleza de la riqueza de las naciones, donde sienta las bases para la ingeniería industrial y en donde el proceso de generación se centra en la optimización de las actividades individuales y la división del trabajo. Para Koskela (2000) los estudios de Smith han constituido, hasta la actualidad, uno de los grandes paradigmas dentro del aumento y generación de efectivo.

En el caso de la logística, los especialistas Mather (1988) y Chistopher (2004) consideran que el aumento del efectivo está directamente relacionado con el concepto de flujo, donde el flujo de materiales y de la información impulsa directamente al flujo de efectivo; es decir, la optimización de la cadena logística crea un aumento en el efectivo.

Haciendo una revisión de las anteriores posiciones sobre el aumento de efectivo (cuadro 1), observamos que existen diversas ideas, muy coherentes, claras, pero parciales, de cómo se realiza el aumento del efectivo dentro de la empresa y de cómo se genera este efectivo. Todas estas concepciones guiadas principalmente por el enfoque de caja negra, donde cada uno de los diferentes puntos de vistas académicos han descartado a los demás sin unificar los criterios, recordemos que el todo es más grande que la suma de sus partes (Mather, 1988), por lo que no comprender en forma integral cómo se aumenta y genera el efectivo de la empresa no da pauta para crear consenso dentro de los integrantes de la empresa que permita orientar las acciones hacia un aumento del efectivo. 


\section{Cuadro 1}

\section{El aumento y generación de efectivo desde diferentes perspectivas}

\begin{tabular}{|c|c|c|c|c|}
\hline \multirow[b]{2}{*}{ Disciplina } & \multirow[b]{2}{*}{$\begin{array}{l}\text { El aumento de } \\
\text { efectivo se logra a } \\
\text { través de: }\end{array}$} & \multicolumn{3}{|c|}{ La generación está dominada por una orientación en: } \\
\hline & & la trasformación & $\begin{array}{c}\text { en el flujo de } \\
\text { materiales e } \\
\text { información }\end{array}$ & $\begin{array}{l}\text { en el valor } \\
\text { añadido }\end{array}$ \\
\hline $\begin{array}{l}\text { Administración } \\
\text { financiera }\end{array}$ & $\begin{array}{l}\text { Inversión financiera, } \\
\text { el financiamiento y } \\
\text { el ahorro }\end{array}$ & $\begin{array}{l}\text { Se da como } \\
\text { consideración de } \\
\text { la naturaleza del } \\
\text { negocio }\end{array}$ & & \\
\hline Economía & $\begin{array}{l}\text { Mejora en las } \\
\text { fuerzas de oferta y } \\
\text { demanda }\end{array}$ & $\begin{array}{l}\text { Interacción trabajo y } \\
\text { capital }\end{array}$ & & \\
\hline Ingeniería & $\begin{array}{l}\text { Optimización de } \\
\text { procesos y aumento } \\
\text { de la productividad }\end{array}$ & $\begin{array}{l}\text { Los procesos } \\
\text { productivos son } \\
\text { la base de la } \\
\text { generación }\end{array}$ & & \\
\hline Mercadotecnia & $\begin{array}{l}\text { Desarrollo de } \\
\text { nuevos mercados, } \\
\text { mejora del portafolio } \\
\text { de productos }\end{array}$ & & & $\begin{array}{l}\text { Acciones de } \\
\text { producto- precio } \\
\text { que sean mejores } \\
\text { que competencia }\end{array}$ \\
\hline Logística & $\begin{array}{l}\text { La administración } \\
\text { de la cadena de } \\
\text { suministro }\end{array}$ & & $\begin{array}{l}\text { Optimización } \\
\text { del flujo de } \\
\text { materiales y de } \\
\text { información }\end{array}$ & \\
\hline $\begin{array}{l}\text { Estrategia } \\
\text { empresarial }\end{array}$ & $\begin{array}{l}\text { Aumento de } \\
\text { estrategias } \\
\text { competitivas }\end{array}$ & & & $\begin{array}{l}\text { Satisfacción del } \\
\text { cliente, ahorro en } \\
\text { costos. }\end{array}$ \\
\hline
\end{tabular}

En el cuadro 1 se observa que algunos académicos han difundido la forma en cómo se aumenta el efectivo sin unificar criterios y, en muchos casos, se ha visto como un hecho que sucede comúnmente dentro de la empresa y que bajo estos diferentes criterios el aumento se puede alcanzar con sólo realizar algunas acciones. Si bien, el aumento de efectivo está claramente ligado con la actividad que desarrolla la empresa, se debe desarrollar un punto de vista que no sea simple, parcial o limitativo, en consecuencia, nuestra siguiente labor será revisar cómo se logra un aumento de efectivo para después enfocarnos en la manera en cómo podemos medirlo.

\section{¿El aumento de efectivo es sólo una medida escalar?}

En nuestro estudio surge una pregunta inicial: ¡el aumento de efectivo es sólo una medida escalar?; esto es, únicamente basta el paso del tiempo y la realización de 
las actividades propias de la empresa para que el efectivo con el cual se inició termine siendo mayor. Si bien el aumento del efectivo está referido al incremento del mismo dentro de un periodo con respecto a un estado inicial, es decir:

$$
\Delta \mathrm{e}_{\mathrm{i}}=\frac{\boldsymbol{e}_{f}-\boldsymbol{e}_{i}}{\mathrm{t}}
$$

Con la condicional de que: $\boldsymbol{e}_{f}>\boldsymbol{e}_{i}$

$\mathrm{e}_{\mathrm{f}} \quad$ efectivo al final del periodo

$\mathrm{e}_{\mathrm{i}} \quad$ efectivo al inicio del periodo

$\mathrm{t}$ periodo de tiempo

$\Delta \mathrm{e}_{\mathrm{i}}$ incremento de efectivo

Entonces si se observa la fórmula anterior, se puede deducir que el paso del tiempo y el trabajo de las unidades productivas dentro de la empresa son suficientes para asegurar un aumento de efectivo; sin embargo, en la práctica, según nuestra experiencia, esto no sucede así. Para lograrlo, se tienen que realizar acciones en el presente para asegurar el incremento de efectivo en el corto, mediano y largo plazos (Morúa, 2009). También es importante asegurar la continuidad de las acciones que permitan la generación de efectivo (Goldratt, 1992).

Así, la anterior fórmula da una pauta inicial para comprender el aumento de efectivo. Iniciaremos por desglosar las diferentes formas en que se puede lograr que el efectivo obtenido al final del periodo sea superior al efectivo inicial. Para esto existen tres principales formas (Morúa, 2009):

1. Disminuir el efectivo gastado, es decir, tomando acciones para ahorrar tanto en costos (de adquisición de las materias primas y de los costos directos) como en gastos.

2. Aumentar el efectivo entrante (ingresos) mediante acciones como el aumento de precio de los productos y servicios que la empresa ofrece, aumento en los ingresos diferentes, aquellos que están relacionados directamente con la actividad de la empresa (inversiones, intereses, etc.) y/o mejorar el proceso de generación.

3. Recuperar efectivo. Adicionalmente a los puntos anteriores, se debe considerar que la empresa maneja efectivo, tanto de entrada como de salida, que están orientados por la operación con actores fuera de la empresa; es 
decir, con clientes, proveedores principalmente. Esta cantidad de efectivo se refiere, por ejemplo, a los créditos otorgados a los clientes, a los créditos otorgados por los proveedores. La condición ideal en el caso de recuperación de efectivo es que el plazo de pago otorgado por parte del proveedor sea igual a la suma del tiempo total de producción del producto más el plazo otorgado al cliente para su pago. De tal forma que los recursos invertidos para producir serían sumamente bajos. Con estas acciones el efectivo recuperado podría utilizarse para reinsertarse dentro del ciclo de generación de efectivo y así aumentaría el efectivo dentro de la empresa.

A su vez, debemos analizar las diferentes fuentes de donde la empresa puede obtener efectivo. Mackevicius (2006) menciona tres principales: a través de inversiones, de acciones de financiamiento y por las operaciones de la misma empresa (proceso de generación). Cabe resaltar que, para la mayoría de la Pyme, la principal fuente de obtención de efectivo es por las operaciones de la empresa misma (Mackevicius, 2006). Entonces para la Pyme, el aumento del efectivo está relacionado principalmente con el proceso de generación de efectivo; es decir, con la actividad preponderante de una empresa, dejando a un lado la forma de incrementarlo a través de inversiones y otras formas financieras por su limitado acceso a los mercados de capitales.

Para Goldratt (1989), es mejor aumentar el efectivo a través del proceso de generación que ahorrar en costos y gastos. Dicho autor, aparte de proponer un sistema de medición y una forma de controlar el piso de producción, crea toda una propuesta de procesos de pensamientos para dirigir las acciones de la empresa hacia el aumento continuo del efectivo a través de su teoría de restricciones. Sin embargo, desde nuestro punto de vista su teoría queda inconclusa al no involucrar los factores humanos, la creación de valor y las diferentes necesidades de flujo de materiales (Morúa, 2009), aunque llega a ser una aproximación muy interesante para desarrollar una continuidad en el aumento y generación de efectivo.

El aumento de efectivo es más que una medición escalar, se convierte principalmente en el resultado de una serie de estrategias que deberán contemplar el factor tiempo y el ciclo de conversión de las materias primas en productos terminados. Nuestra siguiente tarea es analizar la forma en cómo se genera el efectivo dentro de la Pyme de manufactura, lo que nos obliga a revisar, en primer lugar, el ciclo de conversión de efectivo en las empresas, analizando desde el momento que se 
compran los materiales, con cierto plazo de pago, hasta entregarlos al cliente, el cual pagará en un futuro acordado; en segundo lugar, a analizar la forma en qué estas materias primas se convierten en productos terminados.

\section{El ciclo típico de conversión de stocks en empresas de manufactura}

El tiempo ciclo de conversion de stock ha sido estudiado por diferentes autores. Christopher (2004) lo llama tiempo reactor, lo define como la sumatoria del tiempo que pasa desde que se mantienen las materias primas en almacén, se procesan y se conservan en almacen de productos terminados; Mather (1988), tomando como base al sistema de producción toyota, lo define como el tiempo de entrega acumulado de un producto; a su vez Özbayrak (2006) lo llama tiempo de conversión de stocks.

En este artículo, se toma como punto de apoyo el trabajo desarollado por Özbayrak (2006) debido a que define al ciclo de conversión de stocks como la sumatoria de los tiempos ciclo de conversión de materias primas, de conversión de producción en proceso y de conversión del producto terminado. Esta división permitirá determinar los diferentes momentos del ciclo de efectivo.

En la figura 1 se observan cuatro momentos que determinan el inicio de cada uno de los ciclos:

a. La compra y recepción de la materia prima, la cual inicia el tiempo del ciclo de conversión de las materias primas y está influido directamente por las políticas de compras establecidas por la empresa.

b. El despacho de las materias primas al piso de producción inicia el segundo ciclo, donde las materias primas seguirán una serie de procesos hasta convertirse en productos terminados. Este ciclo está influido por la estrategia de planeación y control de manufactura; es decir, si se ha elegido una estrategia pull donde el ciclo se activa por una demanda conocida por parte del cliente, o una estrategia push en la que el proceso se activa por un proceso de planeación por las políticas de tamaño de lote establecidas, de alistamiento y otras consideración de activación de recursos.

c. El tercer ciclo empieza con el almacenamiento de productos terminados; éstos se conservaran hasta iniciar el último momento que es la entrega al cliente. 
Figura 1

Tiempo ciclo de conversión de stock

\begin{tabular}{|c|c|c|c|}
\hline $\begin{array}{l}\text { Compras de } \\
\text { materias } \\
\text { primas }\end{array}$ & $\begin{array}{l}\text { Despacho de materiales al } \\
\text { piso de producción }\end{array}$ & $\begin{array}{l}\text { Materias } \\
\text { primas } \\
\text { convertidas en } \\
\text { productos } \\
\text { terminados }\end{array}$ & $\begin{array}{l}\text { Productos } \\
\text { terminados } \\
\text { entregados } \\
\text { al cliente }\end{array}$ \\
\hline $\begin{array}{l}\text { Tiempo Ciclo de } \\
\text { conversión de las } \\
\text { materias primas }\end{array}$ & $\begin{array}{l}\text { Tiempo Ciclo de conversión de la } \\
\text { producción en proceso }\end{array}$ & \multicolumn{2}{|c|}{$\begin{array}{l}\text { Tiempo Ciclo de } \\
\text { conversión del producto } \\
\text { terminado }\end{array}$} \\
\hline
\end{tabular}

Fuente: Basado en Özbayrak, 2006

Esta división es importante porque configura la logística de la empresa a la vez que permite establecer las estrategias de manufactura dentro de cada uno de los ciclos de conversión, que dependen de la complejidad peculiar de la estructura de cada empresa y que dictan el flujo de los materiales dentro del sistema en cuestiones como esperas, colas, tamaño de lotes, reglas de carga y descarga, etcétera.

Existe otra peculiaridad al considerar el tiempo ciclo de conversión de stock: el nivel de inventarios que se mantiene durante el tiempo dentro de cada uno de los ciclos, una lenta rotación de inventarios y un alto nivel que conlleva a mantener un elevado tiempo ciclo.

Así, observamos que los procesos de transformación hacen posible la conversión de materias primas en productos terminados; además, las estrategias tomadas para determinar el flujo de los materiales mantienen una gran importancia en el tiempo ciclo de conversión de stock, que forma parte del ciclo de conversión de efectivo.

\section{El ciclo típico de conversión de efectivo en empresas de manufactura}

Para una empresa de manufactura el ciclo típico de conversión de efectivo representa el número de días que se requieren para convertir las materias primas en productos terminados, ciclo de conversión de stocks y venderlos a los clientes. Este ciclo incluye los periodos desde el pago de las materias primas hasta el pago de los productos vendidos (Özbayrak, 2006); es decir, incluye la recuperación del efectivo y una parte del financiamiento. 
En la figura 2 se observa el ciclo de conversión de efectivo: las materias primas, que son recibidas por los proveedores, serán usadas en la etapa de producción para su transformación; posteriormente, se almacenarán comúnmente como productos terminados (PT) que serán entregados a los clientes, quienes pagarán el costo de los productos, convirtiéndolo nuevamente en efectivo. Esto revela la importancia de la optimización de este tiempo porque este ciclo crea un efecto multiplicador (Morúa, 2009) que puede llevar a cabo el aumento del efectivo.

\section{Figura 2}

\section{Ciclo de conversión de efectivo}

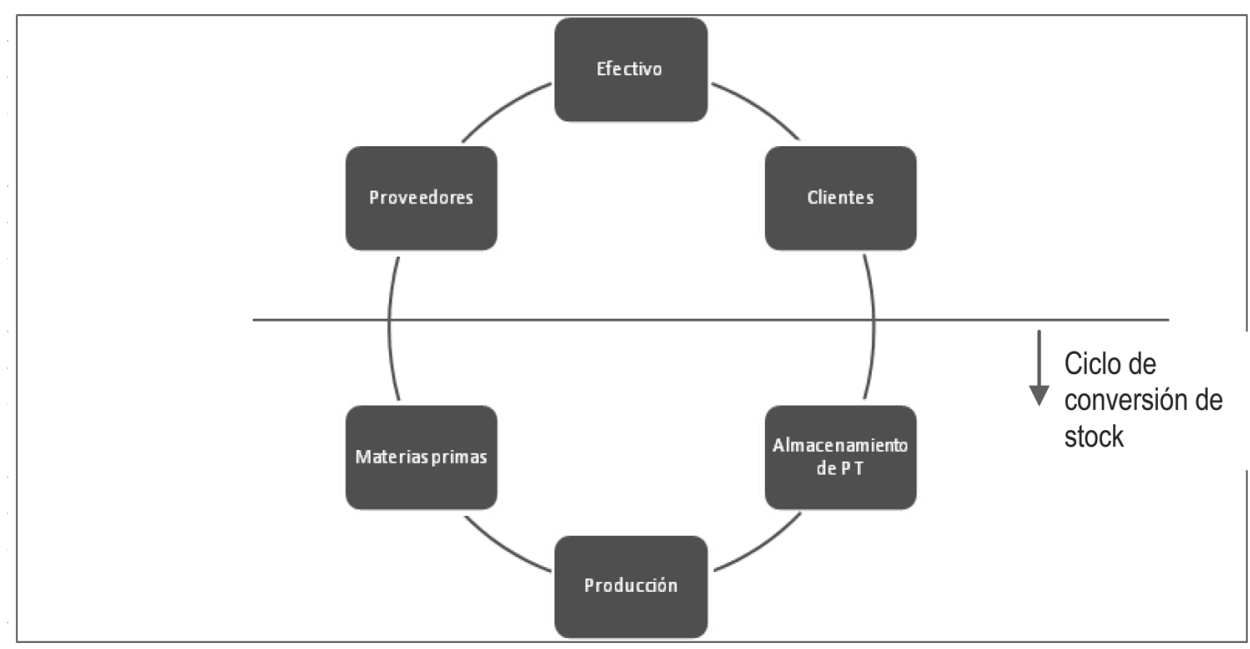

Fuente: Basado en Özbayrak, 2006.

Özbayrak (2006) establece al ciclo de conversión de efectivo como una herramienta importante para el análisis financiero dado que determina el porqué y el cuándo la organización necesita más dinero para sustentar sus actividades, por lo que para la generación de efectivo se requiere tanto de optimizar el ciclo de conversión de stock como optimizar el efectivo que se encuentra fuera del sistema empresa, es decir, con los proveedores y clientes (Morúa, 2009). Además de que el ciclo de conversion de efectivo mantiene una fuerte liga con las políticas de manufactura de las empresas, en particular con la administacion de los niveles de inventarios y con las políticas de control de piso, las cuales dirigen los niveles de materiales desde materias primas, producción en proceso hasta productos terminados (Özbayrak, 2006). 
Observemos entonces que en las empresas de manufactura el ciclo típico de generación de efectivo ${ }^{2}$ se basa en tres consideraciones principales: el proceso de trasformación, un flujo de materiales y la administración de los flujos provenientes de los proveedores y los clientes.

Desde nuestro punto de vista, estos tres elementos no son suficientes para explicar la generación de efectivo. Al hacer un análisis de nuestras experiencias en las empresas mexicanas, compartimos la posición de Savall (2008) quien al realizar un estudio en numerosas empresas determina que existe otro elemento necesario para la generación de efectivo: la creación de valor.

\section{El concepto de valor}

No obstante que es importante el ciclo de conversión de efectivo porque forma parte del aumento del total de efectivo de la empresa y ha ayudado a comprender la necesidad de tres elementos para el aumento de efectivo: la transformación, la administración del flujo de materiales y de efectivo, aun así hace falta un cuarto elemento importante que ha cobrado fuerza en las últimas décadas: el concepto de valor.

Para Cappelletti (2004), el valor es un concepto polisistémico, omnipresente en ciencias de la gestión, cuyo origen proviene de la teoría neoclásica de la economía, que define al valor no como la aportación de los costos, sino por la preferencia de la demanda; es decir, la utilidad marginal de un producto es la que determina su valor; en consecuencia, la demanda resultante de esta utilidad marginal de un bien es el que establece el valor de cambio, por lo que el valor no debe ser percibido como una realidad objetiva fundada en las características del bien o servicio, sino como resultado del comportamiento humano y de sus propias necesidades subjetivas.

Así, el valor al cual nos referimos no es un valor económico establecido por la propia empresa, más bien es el valor subjetivo que mantiene el cliente sobre el producto y servicio que la empresa establece y por el cual está dispuesto a pagar cierta cantidad de dinero. De esta forma, la generación de efectivo se mantiene de

\footnotetext{
${ }^{2}$ Es importante mencionar que el ciclo de conversión de efectivo lo utilizamos para comprender el proceso de generación de efectivo; es decir, la conversión de las materias primas a productos vendidos; por lo tanto, sólo se consideran tanto los gastos provenientes de las acciones de compras de materias primas, como los ingresos generados por este ciclo.
} 
una relación subjetiva, relativa a la atención al cliente, al buscar no sólo satisfacer sus necesidades sino buscar su deleite (Mather, 1992), estableciendo condiciones para que el cliente se pueda mantener por un mayor tiempo como consumidor de los productos y servicios que la empresa ofrece.

\section{Elementos del proceso de generación de efectivo}

El proceso de generación de efectivo regularmente está basado en tres principales ideas interrelacionadas que dominan el pensamiento de los empresarios y directores (Koskela, 2000): la primera basada principalmente en la generación a través del proceso de transformación, la cual ha sido utilizada sin cambios hasta nuestros días, representada en la cadena de valor propuesta por Porter (1985); la segunda referida a el proceso de generación de valor que busca mejorar el valor posible desde el punto de vista del cliente y donde la generación de efectivo se basa en la creación valor en productos, servicios y atención al cliente que mantengan características superiores; la tercera es referida al punto de vista del flujo de materiales donde la importancia del proceso de generación está dominada por la velocidad en que se mueven los materiales y la información.

Lo cierto es que estos tres puntos de vista: trasformación, flujo y valor, con respecto a la generación mantienen una gran interrelación dentro de las organizaciones y al parecer son mutuamente dependientes (Gutiérrez, 2009), por lo que la importancia que requiere cada uno va en relación directa con las necesidades de la empresa. La generación de efectivo se basa en trasformar las materias primas en productos terminados, que éstos sean útiles desde el punto de vista del cliente y que obtenga un valor añadido; además de que el flujo tanto de materiales como de la información relativa al flujo sea optimizado.

El énfasis en cualquiera de estas tres características importantes para la generación (trasformación, flujo y valor) va a depender de las condiciones de la empresa, sus necesidades de efectivo, su posición en el mercado, su portafolios de productos y otras consideraciones; por ejemplo, para una empresa en marcha quizá sea más valioso en un momento dado generar valor y mejorar el flujo de materiales, que otra de nueva creación donde los procesos de trasformación van a dictar muchas veces la supervivencia de la misma.

Goldratt (1992) menciona que la meta de la organización es hacer más efectivo hoy y mañana. Esta idea además de ser clara para todos los integrantes de la em- 
presa involucra a todos los elementos, procesos y recursos dentro de la empresa al ser el proceso de generación un dependiente de estos tres factores, tanto el proceso de transformación que involucra las herramientas técnicas y físicas para elaborar un producto, el valor que este mantenga para un cliente determinado y la entrega oportuna a un costo óptimo interviniendo para este fin toda la cadena logística.

\section{La reconstrucción del aumento de efectivo}

Anteriormente, establecimos la fórmula básica que representa el aumento de efectivo; sin embargo, para poder identificar el incremento de efectivo existen dos tipos de métricas generalmente utilizadas (Commelli, 2007): la posición del efectivo, la cual revela el efectivo disponible al final del periodo; y el análisis del flujo de efectivo que revela la generación dentro del periodo. Así, la propuesta de reconstrucción para afirmar que dentro de un periodo existió un aumento de efectivo, lo estableceremos con las siguientes condiciones: a) deberá existir flujo de efectivo (Commelli, 2007); b) el efectivo total generado deberá de ser mayor al efectivo utilizado para la generación dentro del periodo; c) deberá existir una disposición de efectivo (Commelli, 2007); y d) los inventarios totales finales deberán de ser menores o iguales a los inventarios totales iníciales.

Analicemos cada una de las condiciones anteriores establecidas. El flujo efectivo es referido a la circulación del dinero, éste mide tanto las entradas de efectivo como sus salidas, en tres principales aspectos: el flujo de efectivo de las inversiones, el flujo de efectivo de las actividades de financiamiento y el flujo de efectivo relativo al ciclo de generación (Mackevicius, 2006). Por lo que respecta al efectivo final generado dentro del periodo, éste deberá ser mayor al efectivo inicial, pues es una de las condiciones para que exista un aumento de efectivo dentro del periodo; para lograrlo se deben llevar acciones como: aumento de precios (con sus limitaciones estratégicas que conlleva), estrategias para ahorro, en su amplio sentido, y mecanismos para la recuperación del efectivo. En cuanto a la disposición de efectivo, según Commelli (2007), ella está determinada simplemente por la cantidad de dinero disponible al final del periodo; esta condición lógica se basa en la consideración de que si realmente existe un aumento de efectivo, éste deberá estar disponible para su inmediata operación. Por su parte, los inventarios dentro de las organizaciones representan, entre otras cosas, efectivo invertido; la condición de que los inventarios totales finales sean iguales o menores a los inventaros totales iniciales (Morúa, 2009) establece que la inversión de efectivo con base en los in- 
ventarios no crezca dentro de este periodo; esta condición puede ser modificable dependiendo tanto de cuestiones estratégicas (adquisición planeada de inventarios), por naturaleza de la empresa (empresas que trabajan bajo temporada), por previsión ante un efecto inflacionario donde el efectivo puede perder valor, entre otras establecidas directamente por la dirección de la empresa. Por otro lado, un aumento en las cantidades de inventario representa una disminución del efectivo dentro de la empresa violando directamente la primera (flujo) y tercera condición (disposición).

Contablemente, Brigharn (2006) propone que la prueba del ácido (activo circulante menos inventarios)/pasivo circulante) es una forma de medir la liquidez de la empresa, dando como resultado la cantidad de dinero disponible para hacer frente a las obligaciones a corto plazo. Dentro de esta razón financiera, es importante restar los inventarios porque éstos representan dinero con una alta probabilidad de realización en efectivo, pero aun así no es efectivo. La propuesta de este artículo se basa en el mismo principio, pero es necesario contemplar que los inventarios se deben establecer de manera estratégica porque éstos responden a una forma de amortiguar las variaciones del flujo de materiales; a la forma más común de enfrentar la incertidumbre del mercado; a la manera más fácil de enfrentar los problemas de variabilidad de los procesos que enfrentan las empresas; a la consecuencia más común en los errores de planeación, programación y elaboración de pronósticos (Morúa, 2009); y al resultado de una sobreprotección de los directores y gerentes (Christopher, 2004).

De esta manera, la dualidad que representan los niveles de inventarios, entre lo adecuado para la empresa y lo perjudicial para la misma han dado pauta a estrategias de manufactura que mantienen como filosofía la reducción continua de los mismos.

Integremos los elementos anteriormente vistos para lograr formar la reconstrucción de la forma que se aumenta y genera el efectivo. Para lograrlo, nos apoyaremos en la figura 3 que muestra las cuatro condiciones necesarias para poder afirmar que dentro de un periodo existió un aumento de efectivo. Asimismo, encontramos que el flujo de efectivo va a mantener una relación directa con los procesos de financiamiento, inversión y el proceso de generación, el cual es dependiente de los procesos de transformación relacionados con el ciclo de conversión de stock y del valor agregado como del flujo de materiales y de información. 
Figura 3

El aumento de efectivo

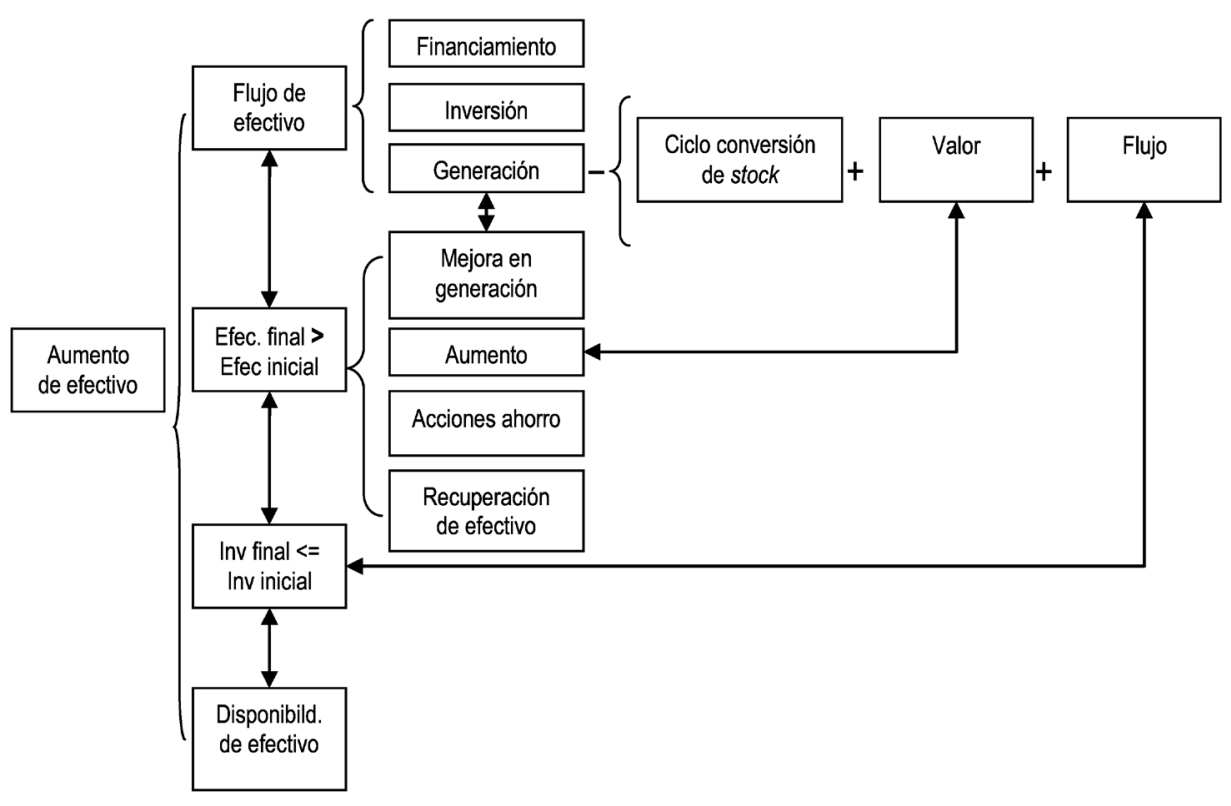

A su vez para lograr que el efectivo final del periodo sea superior al efectivo inicial se observan cuatro acciones relacionadas tanto con el proceso de generación, como con acciones de ahorro, aumento de precios y de recuperación de efectivo.

Como se puede observar el aumento del efectivo está relacionado con cuatro aspectos importantes que marcan la métrica del aumento, los cuales delimitan de manera condicional al aumento: a) la existencia de flujo efectivo dentro de la organización, b) el efectivo final del periodo evaluado sea superior al efectivo inicial, c) disponibilidad de efectivo dentro del periodo, y d) los inventarios iniciales serán menores a los inventarios finales (ésta es una condicional secundaria que deberá ser evaluada dependiendo de la actuación de la empresa y al estado en el que se encuentra). Estas cuatro condiciones marcan necesariamente el hecho de que existe un aumento de efectivo dentro de la organización; por ello las acciones estratégicas y operativas deberán ser guiadas para lograr un aumento de efectivo dentro de la organización.

Con esto no estamos excluyendo las acciones que deben llevar a cabo cada una de las áreas de la empresa como la administración financiera, cuyo objetivo es 
administrar los flujos de efectivo dentro de la empresa, o acciones como los mejoramientos productivos. Más bien lo que proponemos es que cada integrante dentro de la organización comprenda el cómo la empresa puede alcanzar la meta de aumento de efectivo.

\section{Conclusiones y futuras investigaciones}

El estudio del aumento y generación de efectivo ha sido analizado durante mucho tiempo desde una perspectiva de "caja negra" 3 donde uno de los integrantes de las empresas deja a un lado la forma en cómo se aumenta y genera el efectivo, estableciendo bajo sus propias ideas la forma en cómo la empresa deberá trabajar para alcanzar las metas y objetivos.

La propuesta de este artículo consiste en que se utilice el aumento de efectivo como una de las metas principales de la empresa y que se realice su medición evaluando los tiempos de ciclos de generación en sus diferentes etapas; asimismo, es importante que se tomen las acciones correctivas necesarias y se dejen en segundo lugar tanto la medición de la rentabilidad como de la utilidad. Una propuesta similar había sido mencionada por Curtis (1993), al establecer que en la mayoría de las empresas de Japón son medidas primeramente por el flujo de efectivo en lugar de las utilidades generadas debido a que responden más a una condición a largo plazo, permitiendo con esto a las empresas dar más importancia a la velocidad de generación de efectivo y a la realización de relaciones a largo plazo con clientes y proveedores, mediante la administración de la cadena de suministros.

Las estrategias de manufactura cada vez resaltan más la importancia de medir tanto el flujo logístico, flujo de materiales y de información, como al flujo de efectivo, aunque todavía hay pocos trabajos científicos, que integren los aspectos financieros con los físicos para la cadena de suministros (Commelli, 2007).

De esta forma, para enfocarse hacia el aumento y generación de efectivo, se requieren, entre otras cosas: cambios en la forma de calcular los costos de producción (Evia, 2006); políticas, reglas y procedimientos para el establecimiento y manejo de los inventarios; diseño logístico para el manejo adecuado del flujo de materiales

\footnotetext{
${ }^{3}$ En el libro de Knorr (2005) se observa la misma problemática estableciendo que con la familiaridad en el uso de un sistema, regularmente es omitido o ignorado la forma en cómo éste funciona, por lo que los individuos tienden a emplear el enfoque "caja negra".
} 
y de información; estrategias para desarrollar el valor de los productos y servicios; reducción de aquellos factores que limiten el proceso de generación de efectivo.

La aportación teórica de nuestro trabajo contribuye a establecer una serie de futuras investigaciones que se tienen sobre este tema, entre las que se encuentran: elaborar una metodología lógica para mejorar el efectivo dentro de la Pyme, utilizando el enfoque de la teoría trasformación-flujo-valor; establecer las herramientas de control necesarias que permitan realizar tanto un análisis retrospectivo (proceso de control) como también ayudar a la toma de decisiones a través de un pilotaje estratégico (desarrollo de un cuadro de mando integral); y estudiar los elementos que limiten la generación de efectivo para encontrar la forma de reducir sus efectos dentro de la empresa.

\section{Referencias}

Baca, L. (2000). Léxico de la política. México: Flacso.

Baker, M. (2001). Marketing critical perspectives on business and management. Estados Unidos: Routledge.

Brigham, E. y J. Houston (2006). Fundamentos de administración financiera. México: Age Learning Editores.

Cappelletti, L. (2004). Concepts et mesure de la création de valeur organisationnelle. Comptabilité, Contrôle, Audit. Tome 10, Vol.1, juin, 127-146.

Chistopher, M. (2004). Logística: Aspectos estratégicos. México: Limusa.

Comelli, M. (2007). A combined financial and physical flow evaluation for logistics process and tactical production planning: Application in a company supply chain. International Journal of Production Economics. April, 77-95.

Curtis, D. (1993). Making a success in manufacturing. Estados Unidos: McGrawHill Interamericana.

Evia, M. (2006). ¿Es la contabilidad administrativa una herramienta útil para desarrollar la competitividad de las empresas? Contaduría y Administración, UNAM, No. 219, mayo-agosto, 145-166. 
Galbraith, J. (1999). La sociedad opulenta. España: Altaza.

Goldratt, E. y J. Cox (1992). La meta. México: Ediciones Castillo.

Gutiérrez M. Rivera, F., A. Duran y F. Sastrón (2009). Paralelismo entre la teoría de la trasformación-flujo-valor y los tipos de fabricación. Dirección y Organización, No.31 Escuela Politécnica de Madrid, 31-38.

Johnson, R. (2002). Administración financiera. México: Compañía Editorial Continental.

Knorr, K. (2005). La fabricación del conocimiento, un ensayo sobre el cáracter constructivista y contextual de la ciencia. Buenos Aires: Universidad Nacional de Quilmes Editorial.

Koskela, L. (2000). An Exploration Towards a Production Theory and its Application to Construction. Technical Research Center of Finland, VTT Publications 408, Espoo. Disponible en: http://www.inf.vtt.fi/pdf/publications/2000/P408.pdf

Mackevicius, J. (2006). The system of formation and evaluation of the information of cash flows. Journal of Business Economics and Management. Vol. VII, No. 4, 171-182.

Markus, B. y K. Thorbjorn (2003). The role of routines in reducing pervasive uncertainty. Journal of Business Research No. 58. 746 -757.

Mather, H. (1988). Manufactura competitiva. México: Prentice Hall.

Méndez, J. (2002). Economía y empresa. México: McGraw Hill-Interamericana.

Morúa, J. (2009). Guía de estrategias operacionales para incrementar el efectivo en las Pymes. Tesis de Maestría en Ciencias en Ingeniería Industrial. UPIICSA, IPN.

Mula, J. (2006). Models for production planning under uncertainty: A review. International Journal of Production Economics. No. 103, 271-285. 
Özbayrak, M. (2006). The effects of manufacturing control strategies on the cash conversion cycle in manufacturing systems. International Journal of Production Economics, No.103, 535-550.

Ochoa, G. (2002). Administración financiera. México: McGraw-Hill Interamericana.

Porter, M. (1989). Ventaja competitiva. México: CECSA.

Sallenave, J. (1997). La gerencia integral. Colombia: Grupo Editorial Norma.

Savall, H. (2008). Le concept de coût-valeur des activités. Contribution de la théorie socio-économique des organisations. Sciences de Gestion. No. 64. 61-89.

Sink, S. (1999). Planning and measurement in your organization of the future. Industrial Engineering and Management.

Smith, A. (1979). La riqueza de las naciones. México: Fondo de Cultura Económica.

Taylor, J. (2002). Economía. México: Compañía Editorial Continental.

Wegmann G. (1999). Les Tableaux de Bord Stratégiques: Analyse comparative d'un modèle nord-américain et d'un modèle suédois. Disponible en http://panoramix.univ-paris1.fr/GREGOR, consultado el 1 septiembre del 2009.

(A) 